Transactions of the SDPS:

Journal of Integrated Design and Process Science

24 (2), 2020, 1-3

DOI $10.3233 / \mathrm{JID} 200021$

http://www.sdpsnet.org

\title{
EDITORIAL
}

\section{Sustainability: Design, Making, and Technologies}

\author{
Jiami Yang and Yong Zeng* \\ Concordia Institute for Information Systems Engineering, Concordia University, Montreal, Canada
}

Sustainability is the capacity to enable the co-existence of both earth's biosphere and human civilization. The basic premise of sustainability is that the earth's resources cannot be used, depleted, or damaged indefinitely (Portney 2015). Sustainability concerns with three major domains: environment, society, and economy (EPA 2015). Sub-domains of sustainability are cultural, technological, and political (James et al. 2014; Magee et al. 2013). The current strategies for achieving sustainability can generally be divided into three categories: population, affluence, and technology (Holdren, J. P., \& Ehrlich 1974). The most promising path to sustainability is to develop new technologies that address the relationships among the environment, society, and economy, including the design, making, transporting, use, and disposal of the product.

The foremost important task in sustainability is to conduct a holistic analysis of all factors concerned with the environment, society, and economy related to a product. The first paper, authored by Yang et al. and titled "Environment-Based Life Cycle Decomposition (eLCD): Adaptation of EBD to Sustainable Design", proposes a life cycle analysis tool named Environment-based life cycle decomposition (eLCD) to support the sustainable design during the conceptual design stage. In contrast to the existing analysis tools, which focus on the life cycle assessment of a product after the product is already designed or even made, eLCD helps designers effectively and efficiently collect information for sustainability related decisionmaking. In achieving the objective, eLCD decomposes the sustainability environment into three dimensions: environment types, life cycle events, and life cycle time. The environment types are designated as natural, built (including physical artifact and digital artifact), economic, and social environment. It is this holistic environment structure for sustainable conceptual design that makes sustainability is an integral part rather than a burden of the design. The effectiveness of eLCD is demonstrated by its application to the upscaling of a wind turbine, where an energy storage system is introduced to make full use of wind energy with the least waste in serving the electricity demand.

The second paper, authored by Lee et al. and titled "Design Thinking with Appropriate Technology for Improving Social Sustainability: Critical and Comprehensive Criteria", develops criteria for integrating design thinking processes with appropriate technologies for improving social sustainability. The authors argue that current technology promotions are empathetically disconnected from local people and culture due to the lack of affordability, maintenance, and personal training. Five assumptions are proposed for promoting design thinking in the processes: 1) user-oriented design with mass productivity; 2) reiterative nature through user satisfaction surveys; 3 ) affordability for purchase, maintenance, and repair services; 4) local appropriateness; and 5) eco-friendliness with environmental sustainability. They applied the criteria to 28 representative cases from water, energy, health, shelter, and transportation fields. The cases were quantitatively and qualitatively evaluated using a comprehensive literature survey on the use of appropriate

\footnotetext{
* Corresponding author. Email: yong.zeng@concordia.ca.
} 
technologies for the underprivileged. The results show that the criteria are necessary for setting economic, social, and environmental development goals for underprivileged regions.

The third paper is, authored by Demoly and André, is titled "Research Strategy in 4D Printing: Disruptive vs Incremental?". This paper presents 4D printing as a research-intensive technology from a critical external perspective. Most scientific research efforts in 4D printing contribute to developing the spectrum of possible changes by investigating stimulus/smart materials combinations with additive manufacturing technologies. Although the current results are spectacular, the performances are still far from the basic requirements expected in the industry. Therefore, the paper highlights the current limitations and trends towards incremental research strategies and argues that risk-taking and the disruptive nature of research make leaps that benefit society. Based on this paper, it appears that close fields in a situation of possible disciplinary porosity can - with a little openness and some creativity - move away from the current highly self-centered work to try to rekindle 4D printing, provided that risk-taking in interdisciplinary research is better supported. Finally, the paper discusses the possible future of this emerging domain. The technologies discussed in this paper can be a good guidance for this specific kind of technology to serve the making of a sustainable product.

The fourth paper, authored by Hou et al. and titled "An Incentive-Compatible Combinatorial Auction Design for Charging Network Scheduling of Battery Electric Vehicles", proposes an incentive-compatible combinatorial auction for charging network scheduling in a decentralized environment. Charging network scheduling for battery electric vehicles is a challenging research issue on deciding where and when to activate users' charging under the constraints imposed by their time availability and energy demands, limited available capacities, and users' strategic behaviors and untruthful revelation on their real preferences on charging schedules. Hence, it is important to maximize the social welfare across all users, represented by their total values of the scheduled finishing time. The proposed auction mechanism is proved to be individually rational and weakly budget balanced through extensive game-theoretical analysis. Users can bid for their preferred destination and charging time at different stations in such a structured framework. The scheduling specific problem-solving structure is also embedded into the winner determination model to coordinate the charging at multiple stations. They demonstrate its applicability to real-world charging reservation scenarios using the charging network data from Manhattan, New York City. The presented mechanism can be applied to various sharing-economy scenarios.

Sustainability is becoming a more and more important topic in design research. This research relies on design, making, and technologies from diverse communities such as psychology, computer science, engineering, education, and management science. It is a grand challenge for sustainability practice to implement sustainable designs that are more economically and friendly for users, designers, and business owners to achieve their respective objectives. The journal welcomes more reports on experimental and theoretical investigations into the factors that influence innovation and creativity for sustainability. It is a primary objective of this present journal to nurture the growth of transdisciplinary sustainability.

\section{References}

Holdren, J. P., \& Ehrlich, P. R. (1974). Human Population and the Global Environment: Population growth, rising per capita material consumption, and disruptive technologies have made civilization a global ecological force. American Scientist, 62(3), 282-292.

James, P., Magee, L., Scerri, A., \& Steger, M. (2014). Urban sustainability in theory and practice: Circles of sustainability. Urban Sustainability in Theory and Practice: Circles of sustainability. doi:10.4324/9781315765747

Magee, L., Scerri, A., James, P., ... Cahill, F. (2013). Reframing social sustainability reporting: Towards an engaged approach. Environment, Development and Sustainability, 15(1), 225-243.

Portney, K. (2015). Sustainability, MIT Press. Retrieved from https://go.openathens.net/redirector/atsu.edu?url=http $\% 3 \mathrm{~A} \% 2 \mathrm{~F} \% 2 \mathrm{Fwww}$.nature.com $\% 2 \mathrm{Farticles} \% 2$ Fnbt1299_1144.pdf 
EPA. (2015). Sustainability Primer, USA.

\section{Author Biographies}

Jiami Yang is a Research Assistant in the Concordia Institute for Information Systems Engineering at Concordia University. Her major research interest is in design science and its applications to behaviour changes. Particularly, she investigates the impact of mental stress on human performance.

Yong Zeng is a professor in Information Systems Engineering at Concordia University, Montreal. He is the President of Society for Design and Process Science. He was NSERC Chair in aerospace design engineering (2015 - 2019) and Canada Research Chair in design science (2004 - 2014). Zeng researches into creative design by developing and employing mathematical and neurocognitive approaches. He has proposed Environment-Based Design (EBD) addressing the recursive nature of design and the role of mental stress in designer creativity. He applies the EBD to aerospace industry, medical devices, human resource management, municipality, teaching and learning, and health. 